\title{
Preparation and clinical evaluation of a novel lozenge containing polaprezinc, a zinc-L-carnosine, for prevention of oral mucositis in patients with hematological cancer who received high-dose chemotherapy
}

\author{
Hiroko Hayashi $^{1} \cdot$ Ryo Kobayashi $^{1} \cdot$ Akio Suzuki $^{1}$ (D) Yuto Yamada $^{1} \cdot$ \\ Masayuki Ishida $^{1}$ - Toshinobu Shakui ${ }^{2} \cdot$ Junichi Kitagawa $^{3} \cdot$ Hideki Hayashi $^{2}$. \\ Tadashi Sugiyama $^{2} \cdot$ Hirofumi Takeuchi $^{4} \cdot$ Hisashi Tsurumi $^{3} \cdot$ Yoshinori Itoh $^{1}$
}

Received: 9 May 2016/ Accepted: 14 June 2016/Published online: 14 July 2016

(C) The Author(s) 2016. This article is published with open access at Springerlink.com

\begin{abstract}
We previously reported that oral ingestion of polaprezinc, a zinc-L-carnosine, suspended in sodium alginate solution prevents oral mucositis in patients receiving radiotherapy or high-dose chemotherapy. In the present study, we developed a novel preparation of polaprezinc and evaluated clinical effect of the lozenge preparation in patients receiving high-dose chemotherapy for hematopoietic stem cell transplantation. The preparation contained $18.75 \mathrm{mg}$ polaprezinc in a tablet and showed an excellent uniformity and stability up to 24 weeks after storage under room temperature. The incidence rate of grade $\geq 2$ oral mucositis was $74 \%$ in patients without premedication, whereas the rate was remarkably reduced in patients receiving the suspension $(23 \%)$ or lozenge $(13 \%)$ of polaprezinc $(P<0.01)$. The use of non-opioid analgesic drugs such as anti-inflammatory agents and local anesthetics for oral pain was also greatly reduced by polaprezinc suspension or its lozenge (16\% for suspension and $13 \%$ for lozenge compared with $89 \%$ with no premedication, $P<0.01)$. These findings suggest that polaprezinc lozenge is simple to apply and highly effective
\end{abstract}

Akio Suzuki

akio@gifu-u.ac.jp

1 Department of Pharmacy, Gifu University Hospital, 1-1 Yanagido, Gifu 501-1194, Japan

2 Laboratory of Pharmacy Practice and Social Science, Gifu Pharmaceutical University, Gifu, Japan

3 First Department of Internal Medicine, Gifu University Graduate School of Medicine, Gifu, Japan

4 Laboratory of Pharmaceutical Engineering, Gifu Pharmaceutical University, Gifu, Japan for prevention of oral mucositis associated with high-dose chemotherapy for hematopoietic stem cell transplantation.

Keywords Polaprezinc - Lozenge - Oral mucositis ·

Analgesic $\cdot$ High-dose chemotherapy $\cdot$ Hematopoietic stem cell transplantation

\section{Introduction}

Oral mucositis is one of the most common and debilitating complication of cancer treatment, particularly chemotherapy and radiotherapy. This adverse reaction occurs in 20-40\% of patients receiving conventional chemotherapy, in $80 \%$ of patients receiving the conditioning high-dose chemotherapy for hematopoietic stem cell transplant (HSCT), and in almost all patients with head and neck cancer receiving radiotherapy [1-3]. Oral mucositis is often accompanied by pain, odynophagia, dysgeusia and subsequent dehydration and malnutrition, which reduces patients' quality of life (QOL) $[4,5]$. In severe case, discontinuation of therapy or dose reduction is required, which results in the decrease in dose intensity of therapy and reduction in the therapeutic effect $[6,7]$. In addition, the incidence of oral mucositis has negative impact on the healthcare economy requiring costs of care associated with hospitalization, medical management, nutritional support, and management of secondary infection [6, 8]. We also reported that oral mucositis is a significant risk of prolongation of hospital stay in patients with head and neck cancer who received radiotherapy alone or in combination with chemotherapy [9].

Although the precise mechanisms underlying the cytotoxic action of chemotherapy or/and radiotherapy for induction of oral mucosa remain to be clarified, the 
development and healing of oral mucositis are characterized by the following processes: (1) initiation of mucosal injury due to the production of reactive oxygen species, (2) tissue injury and cell death induced by upregulation of proinflammatory cytokines such as tumor necrosis factor (TNF)- $\alpha$, interleukin (IL)-1 and IL-6, (3) formation of mucosal ulceration caused by infiltration of inflammatory cells and worsening of symptoms due to secondary infection, and (4) healing by epithelial proliferation as well as cellular and tissue differentiation [10].

Several agents, including benzydamine [11, 12], sucralfate [13], prostaglandin E2 [14], glutamine [15], granulocyte-macrophage colony-stimulating factor [16], palifermin [17-19], and amifostine [20], have been reported for prevention of oral mucositis; however, most of them, except for palifermin, have no consistent effect. We recently reported that oral ingestion of polaprezinc, a zincL-carnosine, suspended in sodium alginate solution was highly effective for prevention of oral mucositis associated with radiotherapy in patients with head and neck cancer as well as in patients with high-dose chemotherapy for HSCT $[21,22]$. However, there were several drawbacks in the preparation: (1) preparation of polaprezinc suspension is time-consuming, (2) the ingredient is rapidly separated from sodium alginate solution, (3) dosing is not accurate due to the high viscosity of the suspension, and (4) oral acceptability is limited in some patients due to unfavorable taste and undesirable texture. To overcome these problems of the suspension, we have developed a polaprezinc lozenge and evaluated the clinical effect of the preparation for prevention of oral mucositis in patients who received conditioning high-dose chemotherapy for HSCT.

\section{Patients and methods}

\section{Materials}

Polaprezinc (Promac ${ }^{\circledR}$ granules $15 \%$ ) was purchased from Zeria Pharmaceutical Co. Ltd. (Tokyo, Japan). Sodium alginate (KIMICA Algin I-1 ${ }^{\circledR}$, Kimica Co. Ltd., Osaka, Japan), magnesium Stearate (Magnesium Stearate ${ }^{\circledR}$, Mallinckrodt Japan Co. Ltd., Tokyo), acesulfame potassium (Sunett ${ }^{\circledR}$ Pharma Grade Type I, MC Food Specialties Inc., Tokyo), aspartame (Aspartame ${ }^{\circledR}$, Ajinomoto Co., Inc., Tokyo), mannitol (Parteck M100 ${ }^{\circledR}$, Merck Ltd., Tokyo), microcrystalline cellulose (CEOLUS UF-711 ${ }^{\circledR}$, Asahi Kasei chemicals Corp., Tokyo), cornstarch (PC-10 ${ }^{\circledR}$, Asahi Kasei chemicals Corp., Tokyo), fragrance material (dry coat ${ }^{\circledR}$, Takata Koryo CO., LTD., Hyogo, Japan) were obtained from commercial sources and used as base materials of the lozenge preparation.

\section{Preparation of polaprezinc lozenge for oral application}

The compositions of polaprezinc lozenge are shown in Table 1 . The mixture was directly compressed with $15 \mathrm{kN}$ by using a single punch tablet press (TAB ALL N-30E Type ${ }^{\circledR}$, Okada Seiko Co. Ltd., Tokyo). One tablets of the lozenge contained $18.75 \mathrm{mg}$ polaprezinc. The thickness and diameter of the preparation were 5.8 and $16.3 \mathrm{~mm}$, respectively. For application of patients, one piece of polaprezinc lozenge was sucked and swallowed for 4 times in a day.

\section{Preparation of polaprezinc sodium alginate suspension}

Polaprezinc $(75 \mathrm{mg}$ ) was suspended in $20 \mathrm{~mL}$ of $5 \%$ sodium alginate solution. A $5 \mathrm{~mL}$ portion of the suspension was orally rinsed for $2 \mathrm{~min}$ and then swallowed for 4 times in a day and continued until 1 month after transplantation.

\section{Determination of polaprezinc}

The content uniformity of the polaprezinc lozenge was tested in 10 separate preparations. Polaprezinc was determined by HPLC with spectrophotometric detection. Sample preparation for HPLC analysis was carried out by the following methods: the tablet was accurately weighted and finely pulverized, and the resultant powder was suspended in $10 \mathrm{~mL}$ of $0.1 \mathrm{M}$ hydrochloric acid solution. Then, the suspension was centrifuged at $2000 \times g$ for $5 \mathrm{~min}$. The supernatant was filtrated with $0.2-\mu \mathrm{m}$ filter and used for analysis. A $10-\mu 1$ portion of the filtrated supernatant was directly injected onto HPLC.

The HPLC system consisted of a separation column (Shim-pack FC-ODS; $150 \mathrm{~mm} \times 4.6 \mathrm{~mm}$ in diameter, $5 \mu \mathrm{m}$ of sphere size, Shimadzu, Kyoto, Japan), column oven whose temperature was set at $40{ }^{\circ} \mathrm{C}$, and spectrophotometric detector (SPD-10A, Shimadzu). The mobile

Table 1 Composition of polaprezinc lozenge in one tablet

\begin{tabular}{lc}
\hline Polaprezinc & $18.75 \mathrm{mg}$ \\
Sodium alginate & $0.05 \mathrm{~g}$ \\
Magnesium stearate & $0.005 \mathrm{~g}$ \\
Acesulfame potassium & $0.0015 \mathrm{~g}$ \\
Aspartame & $0.0015 \mathrm{~g}$ \\
Mannitol & $0.33 \mathrm{~g}$ \\
Cellulose & $0.4 \mathrm{~g}$ \\
Cornstarch & $0.05 \mathrm{~g}$ \\
Fragrance material & $0.01 \mathrm{~g}$ \\
\hline
\end{tabular}


phases were $10 \mathrm{mM}$ phosphate buffer at $\mathrm{pH} 3.5$ (A) and acetonitrile (B). The HPLC analysis was carried out by using a gradient elution with the following method: the concentration of mobile phase B was started at $10 \%$ from 0 to $2 \mathrm{~min}$, then, the linear gradient from 10 to $50 \%$ applied from 2 to $4 \mathrm{~min}$ and increased at $50 \%$ from 4 to 6 min. Thereafter, the acetonitrile concentration was lowered to $10 \%$ from 6 to $7 \mathrm{~min}$ and maintained the same composition for further $3 \mathrm{~min}$. Polaprezinc was detected at $210 \mathrm{~nm}$.

\section{Uniformity of polaprezinc lozenge preparation}

The acceptance value (AV) of the preparation is less than $15 \%$, according the Japanese Pharmacopoeia 16th edition (JP16). AV for JP 16 was calculated according to the following equation: $\mathrm{AV}=|M-X|+k s$, in which $M$ is reference value, $X$ is the average of individual contents expressed as the percentage of the label claim, $k$ is acceptability constant, and $s$ represents sample standard deviation.

\section{Stability test}

A portion of the preparation was stored at $25^{\circ} \mathrm{C}$ with $50-60 \%$ humidity (normal condition) or at $40{ }^{\circ} \mathrm{C}$ with $75 \%$ humidity (accelerated condition) for 2-24 weeks, and then the content of polaprezinc was determined. Another set of test was performed to determine the hardness of the stored preparation.

\section{Evaluation of clinical effect of polaprezinc lozenge for prevention of oral mucositis in patients with conditioning high-dose chemotherapy for HSCT}

Patients were pretreated with either polaprezinc suspension during January 2013 and December $2014(N=31)$ or polaprezinc lozenge during January 2015 and December 2015 ( $N=16)$ for prevention of oral mucositis. Data were compared between the two groups. Moreover, the data obtained from patients who received high-dose chemotherapy without any premedication during March 2006 and February 2011 were used as negative control.

The incidence and severity of oral mucositis and its associated symptoms such as oral pain and other adverse events were reviewed from medical records and compared among three groups. The severity of adverse events was graded according to the Common Terminology Criteria for Adverse Events (CTCAE) version 3.0. The prevalence of the use of non-opioid analgesics, including anti-inflammatory drugs and local anesthetics, and opioid analgesics was also compared.

\section{Ethical approval}

This study was carried out in accordance with the guidelines for human studies adopted by the ethics committee of the Gifu University Graduate School of Medicine and notified by the Japanese government (Institutional review board approval No. 25-79). All participants except for patients who received high-dose chemotherapy without any premedication provided written informed consent prior to participation.

\section{Statistical analysis}

Data were analyzed by using IBM SPSS statistics version 22(IBM Japan Services Co., Ltd., Tokyo). The incidence rates of adverse events were statically compared among three groups by Kruskal-Wallis test followed by Dunn's test or Scheffe's test for multiple comparison. Parametric variables were analyzed by one-way analysis of variance followed by Scheffe's test. $P$ values of $<0.05$ were considered statically significant.

\section{Results}

The average of polaprezinc content in 10 preparation of polaprezinc lozenge was $19.6+0.52 \mathrm{mg}$, and the values were ranging from 101.6 to $108.7 \%$. The relative standard deviation was $2.8 \%$. Thus, $A V$ was $9.2 \%$, a value that was within the limit (15\%) of uniformity of dosage units for JP 16.

When the polaprezinc lozenge was stored in polyethylene package under normal condition for 24 weeks, no apparent changes in the polaprezinc content, form, or color of preparation were observed. The contents of polaprezinc were fairly stable ranging $99.8 \%$ to $102.4 \%$ during 24 weeks after storage at $25{ }^{\circ} \mathrm{C}$ with 50-60\% humidity (normal condition) (Fig. 1). However, a slight change in color from white to light brown appeared in the preparation stored for 24 weeks in a chamber controlled at $40{ }^{\circ} \mathrm{C}$ and $75 \%$ in humidity (accelerated condition), although the contents of polaprezinc were almost stable (Fig. 1).

The demographics of patients receiving no premedication, premedication with either suspension or lozenge of polaprezinc are shown in Table 2. There were no significant differences in gender, age, laboratory data, type of leukemia and the rate of regimen containing Ara-C or MTX among three groups.

The polaprezinc lozenge preparation stored at normal condition for up to 12 weeks was used in the present clinical study. As shown in Fig. 2, the incidence rates of grade 2 and grade 3 oral mucositis were 73.7 and $21.1 \%$, respectively, in no premedication control group, while the 


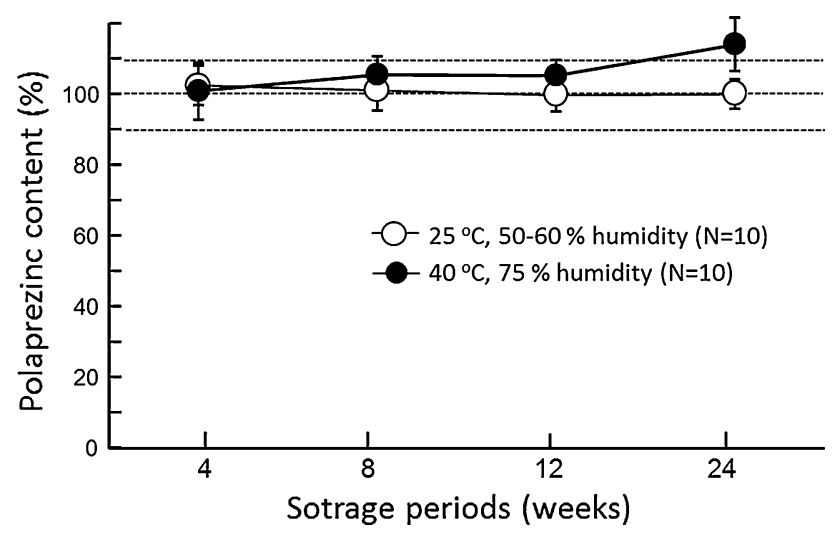

Fig. 1 Stability of polaprezinc lozenge after storage under normal condition (A) or accelerated condition (B) for up to 24 weeks. Each tablet was wrapped in an aluminum package and stored at $25^{\circ} \mathrm{C}$ with $50-60 \%$ humidity (normal condition) or at $40{ }^{\circ} \mathrm{C}$ with $75 \%$ humidity (accelerated condition). Each column represents the mean \pm SD of 10 experiments

rates were greatly reduced in polaprezinc suspension group (22.6\% for grade $2, P<0.01$ by Kruskal-Wallis test followed by Scheffe's test, and $3.2 \%$ for grade $3, \mathrm{NS}$ ) and polaprezinc lozenge group $(12.5 \%$ for grade $2, P<0.01$, and $6.3 \%$ for grade $3, \mathrm{NS}$ ). The average grade of oral mucositis was 0.6 for suspension group as well as for lozenge group, both of which were significantly lower than that (1.7) for control group $(P<0.01$ by Kruskal-Wallis test followed by Dunn's test). On the other hand, there was no significant difference in the average grade or incidence rate of oral mucositis between suspension group and lozenge group.

Similarly, the incidence rates of moderate to severe pain were also markedly reduced in suspension group and lozenge group as compared with control group. As a consequence, the prevalence for the use of non-opioid analgesics markedly decreased in suspension group $(16.1 \%$, $P<0.01$, for suspension group; $12.5 \%, P<0.01$, for lozenge group), compared with control group $(89.5 \%)$, although the prevalence for the use of opioid analgesics was not significantly different among three groups.

As shown in Table 3, there were no significant differences in the incidence rates of other adverse events such as rash, pruritus, erythema, nausea, vomiting, and febrile neutropenia among there groups.

\section{Discussion}

The present newly developed polaprezinc lozenge met the criteria of uniformity defined as $<15 \%$ of $\mathrm{AV}$ in the dosage uniformity by JP 16 . This preparation was stable in

Table 2 Patient demographics and clinical characteristics

\begin{tabular}{|c|c|c|c|c|}
\hline & No premedication & Polaprezinc suspension & Polaprezinc lozenge & $P$ value \\
\hline Number of patients (male/female) & $19(13 / 6)$ & $31(24 / 7)$ & $16(10 / 6)$ & $0.755^{\mathrm{a}}$ \\
\hline Age (medium, range) & $49.2(26-73)$ & $54.5(19-73)$ & $55.8(22-70)$ & $0.370^{\mathrm{b}}$ \\
\hline Serum albumin $(g / d L)$ & $3.9 \pm 0.5$ & $3.7 \pm 0.5$ & $3.9 \pm 0.4$ & $0.370^{\mathrm{c}}$ \\
\hline Aspartate transaminase (U/L) & $30.8 \pm 28.6$ & $28.3 \pm 26.4$ & $23.1 \pm 9.5$ & $0.643^{\mathrm{c}}$ \\
\hline Alanine aminotransferase (U/L) & $25.8 \pm 15.5$ & $31.6 \pm 32.8$ & $23.6 \pm 18.3$ & $0.543^{\mathrm{c}}$ \\
\hline Serum creatinine $(\mathrm{mg} / \mathrm{dL})$ & $0.87 \pm 0.77$ & $0.72 \pm 0.19$ & $0.62 \pm 0.14$ & $0.235^{\mathrm{c}}$ \\
\hline White blood cells $\left(/ \mathrm{mm}^{3}\right)$ & $5534 \pm 5010$ & $4162 \pm 2928$ & $4389 \pm 3030$ & $0.734^{\mathrm{c}}$ \\
\hline Hemoglobin (g/dL) & $10.5 \pm 2.3$ & $10.1 \pm 2.0$ & $10.8 \pm 2.4$ & $0.549^{c}$ \\
\hline Platelet $\left(/ \mathrm{mm}^{3}\right)$ & $15.4 \pm 7.1$ & $17.1 \pm 14.4$ & $20.4 \pm 14.9$ & $0.515^{\mathrm{c}}$ \\
\hline Diagnosis $(\%)$ & & & & $0.487^{\mathrm{a}}$ \\
\hline Acute myeloid leukemia & $1(5.3)$ & $10(32.3)$ & $5(31.3)$ & \\
\hline Acute lymphoid leukemia & $8(42.1)$ & $1(3.2)$ & $3(18.8)$ & \\
\hline Acute promyelocytic leukemia & 0 & $1(3.2)$ & 0 & \\
\hline Myelodysplastic syndromes & $1(5.3)$ & $2(6.4)$ & 0 & \\
\hline NK/T cell lymphoma & & 7 (22.6) & $2(12.5)$ & \\
\hline Diffuse large B cell lymphoma & $5(26.3)$ & $8(25.8)$ & $5(31.3)$ & \\
\hline Mantle cell lymphoma & $1(5.3)$ & 0 & $1(6.3)$ & \\
\hline Follicular lymphoma & 0 & $1(3.2)$ & 0 & \\
\hline Hodgkin lymphoma & $1(5.3)$ & $1(3.2)$ & 0 & \\
\hline Chemotherapy regimen (\%) & & & & $0.730^{\mathrm{a}}$ \\
\hline Ara-C-based regimen & 7 (38.9) & $16(51.6)$ & $10(62.5)$ & \\
\hline MTX-based regimen & $11(61.1)$ & $15(35.5)$ & $6(25.0)$ & \\
\hline
\end{tabular}

${ }^{\mathrm{a}} m \times n$ Chi-square test, ${ }^{\mathrm{b}}$ Kruskal-Wallis test, ${ }^{\mathrm{c}}$ one-way ANOVA followed by Scheffe test 
Fig. 2 Comparison of effects of polaprezinc suspension and lozenge on the incidence and grade of oral mucositis and prevalence of the use of analgesics in patients receiving conditioning high-dose chemotherapy for stem cell hematopoietic stem cell transplantation. $\dagger P<0.01$ versus control by KruscalWallis test followed by Dunn's test, $* P<0.01$ versus control by Kruscal-Wallis followed by Scheffe's test. No significant difference in each parameter was observed between suspension and lozeng

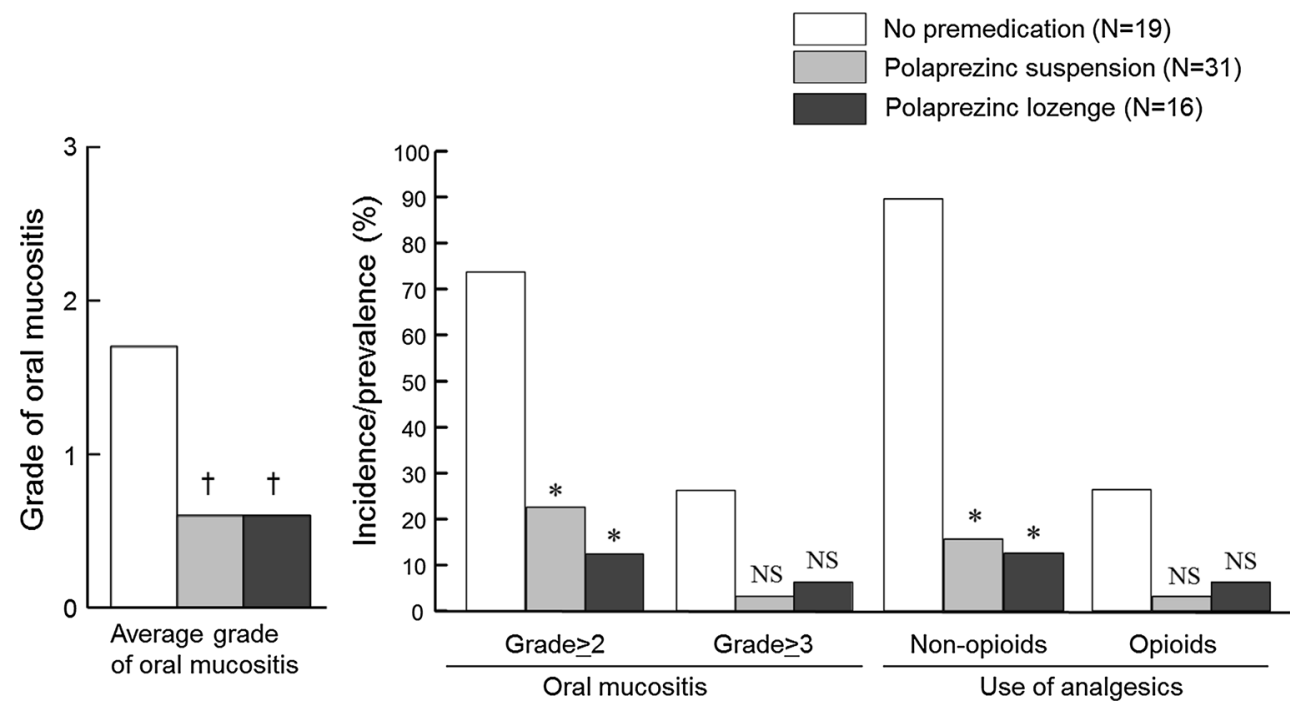

\begin{tabular}{lcccc}
\hline & No premedication & Polaprezinc suspension & Polaprezinc lozenge & $P$ value \\
\hline Rash & $6(31.6)$ & $13(41.9)$ & $4(25.0)$ & 0.109 \\
Pruritus & $6(31.6)$ & $8(25.8)$ & $3(18.7)$ & 0.875 \\
Erythema & $6(31.6)$ & $11(35.5)$ & $6(37.5)$ & 0.991 \\
Nausea (grade $>2)$ & $6(31.6)$ & $12(38.7)$ & $13(81.3)$ & 0.493 \\
Vomiting & $6(31.6)$ & $3(9.7)$ & $2(12.6)$ & 0.257 \\
Febrile neutropenia & $18(94.7)$ & $25(80.6)$ & $13(81.3)$ & 0.624
\end{tabular}

Kruskal-Wallis test followed by Scheffe test
Table 3 Comparison of the incidence of other nonhematological adverse events among patients receiving nonpremedication, suspension and lozenge preparations of polaprezinc
TNF- $\alpha$ and IL-6 in the oral mucosa after exposure to highdose chemotherapy is considered to be implicated in the pathogenesis [23, 24]. Polaprezinc is currently used as antiulcer drug. It contains zinc, an essential trace element used for the therapy of gastric ulcer [25], in its molecule. It has been demonstrated that polaprezinc has protective action on the mucosal cells against noxious stimuli [26].The anti-oxidant action is considered to contribute to the mucoprotective action of polaprezinc [27, 28]. It has also been demonstrated that the mucoprotective action of polaprezinc is mediated at least in part by the enhancement of the expression of hemeoxygenase (HO)-1 [29]. Naito et al. [30] reported that polaprezinc reverses aspirin-induced increases in lipid peroxidation, neutrophil accumulation, and TNF- $\alpha$ expression in rat gastric mucosa. On the other hand, Wada et al. reported that enhancement of the expression of $72-\mathrm{kDa}$ heat shock protein, an endogenous cytoprotective factor, plays an important role in the mucoprotective action of polaprezinc [31]. Therefore, it is highly likely that the antioxidative action of polaprezinc may contribute to the preventive effect of this compound against oral mucositis.

The clinical practice guidelines for the management of mucositis secondary to cancer therapy promulgated by the oxygen species and proinflammatory cytokines such as 
Multinational Association of Supportive Care in Cancer (MASCC)/International Society of Oral Oncology (ISOO) suggest that oral ingestion of zinc supplements may be of benefit to prevent oral mucositis in patients receiving radiotherapy or chemotherapy [23].

Currently, cancer chemotherapy is shifting from inpatient admission to outpatients setting. However, some of cytotoxic anti-cancer drugs or molecularly targeted drugs for the treatment of cancer in application to outpatients frequently develop an oral mucositis. Polaprezinc lozenge is a solid portable dosage form and can be easily and safely taken without water. Thus, it is highly probable that the present lozenge preparation is applicable not only to hospitalized patients but also to those in the ambulatory chemotherapy setting.

In conclusion, we newly developed the lozenge containing polaprezinc for prevention of oral mucositis. The preparation showed an excellent uniformity and stability. The polaprezinc lozenge was highly effective for prevention of moderate to severe oral mucositis in patients receiving high-dose chemotherapy for HSCT. The efficacy of the lozenge preparation was almost comparable to that of polaprezinc suspension in sodium alginate. Both the lozenge and suspension also reduced the occurrence of accompanying oral pain to the similar extent. Therefore, it is suggested that the present polaprezinc lozenge preparation is potentially useful for prevention of oral mucositis in cancer patients who receive high-dose chemotherapy.

Acknowledgments This work was supported in part by a Grant-inAid for Scientific Research (C25460208) from the Ministry of Education, Science, Sport and Culture, Japan.

\section{Compliance with ethical standards}

Conflict of interest All authors declare that they have no conflicts of interest concerning this work.

Open Access This article is distributed under the terms of the Creative Commons Attribution 4.0 International License (http://crea tivecommons.org/licenses/by/4.0/), which permits unrestricted use, distribution, and reproduction in any medium, provided you give appropriate credit to the original author(s) and the source, provide a link to the Creative Commons license, and indicate if changes were made.

\section{References}

1. Jones JA, Avritscher EB, Cooksley CD, Michelet M, Bekele BN, Elting LS. Epidemiology of treatment-associated mucosal injury after treatment with newer regimens for lymphoma, breast, lung, or colorectal cancer. Support Care Cancer. 2006;14(6):505-15.

2. Vera-Llonch M, Oster G, Hagiwara M, Sonis S. Oral mucositis in patients undergoing radiation treatment for head and neck carcinoma. Cancer. 2006;106(2):329-36.

3. Vera-Llonch M, Oster G, Ford CM, Lu J, Sonis S. Oral mucositis and outcomes of allogeneic hematopoietic stem-cell transplantation in patients with hematologic malignancies. Support Care Cancer. 2007;15(5):491-6.

4. McGuire DB, Altomonte V, Peterson DE, Wingard JR, Jones RJ, Grochow LB. Patterns of mucositis and pain in patients receiving preparative chemotherapy and bone marrow transplantation. Oncol Nurs Forum. 1993;20(10):1493-502.

5. Duncan GG, Epstein JB, El TuD, Sayed S, Bezjak A, Ottaway J, et al. National Cancer Institute of Canada Clinical Trials Group. Quality of life, mucositis, and xerostomia from radiotherapy for head and neck cancers: a report from the NCIC CTG HN2 randomized trial of an antimicrobial lozenge to prevent mucositis. Head Neck. 2005;27(5):421-8.

6. Elting LS, Cooksley C, Chambers M, Cantor SB, Manzullo E, Rubenstein EB. The burdens of cancer therapy. Clinical and economic outcomes of chemotherapy-induced mucositis. Cancer. 2003;98(7):1531-9.

7. Trotti A, Bellm LA, Epstein JB, Frame D, Fuchs HJ, Gwede CK, et al. Mucositis incidence, severity and associated outcomes in patients with head and neck cancer receiving radiotherapy with or without chemotherapy: a systematic literature review. Radiother Oncol. 2003;66(3):253-62.

8. Elting LS, Cooksley CD, Chambers MS, Garden AS. Risk, outcomes, and costs of radiation-induced oral mucositis among patients with head-and-neck malignancies. Int $\mathrm{J}$ Radiat Oncol Biol Phys. 2007;68(4):1110-20.

9. Suzuki A, Kobayashi R, Okayasu S, Kuze B, Aoki M, Mizuta K, et al. Pharmacotherapy for adverse events reduces the length of hospital stay in patients admitted to otolaryngology ward: a single arm intervention study. PLoS One. 2014;9(12):e115879. doi:10. 1371/journal.pone.0115879 (eCollection 2014).

10. Lalla RV, Sonis ST, Peterson DE. Management of oral mucositis in patients who have cancer. Dent Clin N Am. 2008;52(1):61-77.

11. Kazemian A, Kamian S, Aghili M, Hashemi FA, Haddad P. Benzydamine for prophylaxis of radiation-induced oral mucositis in head and neck cancers: a double-blind placebo controlled randomized clinical trial. Eur J Cancer Care. 2009;18(2):174-8.

12. Nicolatou-Galitis O, Sarri T, Bowen J, Di Palma M, Kouloulias VE, Niscola P, Riesenbeck D. Systematic review of anti-inflammatory agents for the management of oral mucositis in cancer patients. Support Care Cancer. 2013;21:3179-89.

13. Carter DL, Hebert ME, Smink K, Leopold KA, Clough RL, Brizel DM. Double blind randomized trial of sucralfate vs placebo during radical radiotherapy for head and neck cancers. Head Neck. 1999;21(8):760-6.

14. Labar B, Mrsić M, Pavletić Z, Bogdanić V, Nemet D, Aurer I, et al. Prostaglandin E2 for prophylaxis of oral mucositis following BMT. Bone Marrow Transplant. 1993;11(5):379-82.

15. Tsujimoto T, Yamamoto $Y$, Wasa M, Takenaka Y, Nakahara S, Takagi $\mathrm{T}$, et al. L-glutamine decreases the severity of mucositis induced by chemoradiotherapy in patients with locally advanced head and neck cancer: a double-blind, randomized, placebocontrolled trial. Oncol Rep. 2015;33(1):33-9. doi:10.3892/or. 2014.3564.

16. Masucci G, Broman P, Kelly C, Lindahl S, Malmberg L, Reizenstein J, et al. Therapeutic efficacy by recombinant human granulocyte/monocyte-colony stimulating factor on mucositis occurring in patients with oral and oropharynx tumors treated with curative radiotherapy: a multicenter open randomized phase III study. Med Oncol. 2005;22(3):247-56.

17. Vadhan-Raj S, Trent J, Patel S, Zhou X, Johnson MM, Araujo D, et al. Single-dose palifermin prevents severe oral mucositis during multicycle chemotherapy in patients with cancer: a randomized trial. Ann Intern Med. 2010;153(6):358-67. doi:10.7326/ 0003-4819-153-6-201009210-00003.

18. Henke M, Alfonsi M, Foa P, Giralt J, Bardet E, Cerezo L, et al. Palifermin decreases severe oral mucositis of patients undergoing 
postoperative radiochemotherapy for head and neck cancer: a randomized, placebo-controlled trial. J Clin Oncol. 2011;29(20):2815-20. doi:10.1200/JCO.2010.32.4103.

19. Le QT, Kim HE, Schneider CJ, Muraközy G, Skladowski K, Reinisch S, et al. Palifermin reduces severe mucositis in definitive chemoradiotherapy of locally advanced head and neck cancer: a randomized, placebo-controlled study. J Clin Oncol. 2011;29(20):2808-14. doi:10.1200/JCO.2010.32.4095.

20. Gu J, Zhu S, Li X, Wu H, Li Y, Hua F. Effect of amifostine in head and neck cancer patients treated with radiotherapy: a systematic review and meta-analysis based on randomized controlled trials. PLoS ONE. 2014;9(5):e95968. doi:10.1371/journal. pone.0095968.

21. Watanabe T, Ishihara M, Matsuura K, Mizuta K, Itoh Y. Polaprezinc prevents oral mucositis associated with radiochemotherapy in patients with head and neck cancer. Int J Cancer. 2010;127(8): 1984-90.

22. Hayashi H, Kobayashi R, Suzuki A, Ishihara M, Nakamura N, Kitagawa J, et al. Polaprezinc prevents oral mucositis in patients treated with high dose chemotherapy followed by hematopoietic stem cell transplantation. Anticancer Res. 2014;34(12):7271-7.

23. Lalla RV, Bowen J, Barasch A, Elting L, Epstein J, Keefe DM, et al. MASCC/ISOO clinical practice guidelines for the management of mucositis secondary to cancer therapy. Cancer. 2014;120(10):1453-61.

24. Sonis ST. New thoughts on the initiation of mucositis. Oral Dis. 2010;16(7):597-600. doi:10.1111/j.1601-0825.2010.01681.x.

25. Dajani EZ, Klamut MJ. Novel therapeutic approaches to gastric and duodenal ulcers: an update. Expert Opin Investig Drugs. 2000;9(7):1537-44.
26. Hill TL, Blikslager AT. Effect of a zinc L-carnosine compound on acid-induced injury in canine gastric mucosa ex vivo. Am J Vet Res. 2012;73(5):659-63.

27. Yoshikawa T, Naito Y, Tanigawa T, Yoneta T, Kondo M. The antioxidant properties of a novel zinc-carnosine chelate compound, N-(3-aminopropionyl)-L-histidinato zinc. Biochim Biophys Acta. 1991;1115(1):15-22.

28. Ineu RP, Oliveira CS, Oliveira VA, Moraes-Silva L, da Luz SC, Pereira ME. Antioxidant effect of zinc chloride against ethanolinduced gastrointestinal lesions in rats. Food Chem Toxicol. 2013;58:522-9. doi:10.1016/j.fct.2013.05.022.

29. Ueda K, Ueyama T, Oka M, Ito T, Tsuruo Y, Ichinose M. Polaprezinc (Zinc L-carnosine) is a potent inducer of anti-oxidative stress enzyme, heme oxygenase (HO)-1—a new mechanism of gastric mucosal protection. J Pharmacol Sci. 2009;110(3):285-94.

30. Naito Y, Yoshikawa T, Yagi N, Matsuyama K, Yoshida N, Seto $\mathrm{K}$, et al. Effects of polaprezinc on lipid peroxidation, neutrophil accumulation, and TNF-alpha expression in rats with aspirin-induced gastric mucosal injury. Dig Dis Sci. 2001;46(4):845-51.

31. Odashima M, Otaka M, Jin M, Wada I, Horikawa Y, Matsuhashi $\mathrm{T}$, et al. Expression of HSP72 in the gastric mucosa is regulated by gastric acid in rats-correlation of HSP72 expression with mucosal protection. Biochem Biophys Res Commun. 2006; 349(2):611-8. 